\title{
Synthèse cartographique des connaissances en crues observables dans le Sud-Est de la France
}

\author{
Dominique Faure*, Gilles Galea, Nicole Mathys, Guy Oberlin \\ Cemagref, Lyon et Grenoble
}

Jean-Louis Edouard, Huguette Vivian

Lama, URA 344 CNRS

\section{Introduction}

Les études d'aménagement hydraulique d'un site sont étroitement liées aux risques d'inondations et, plus précisément, aux caractéristiques de crues de fréquences données : débit, durée, hauteur d'eau.

Lorsqu'une station de mesures hydrométriques correspond au site étudié, il est possible, si elle a fonctionné suffisamment longtemps, de procéder à une analyse statistique des relevés historiques et d'évaluer les risques encourus.

Malheureusement ce n'est pas toujours le cas. Lorsque l'estimation directe n'est pas possible au site, il est donc important de pouvoir disposer de méthodes d'évaluations sommaires, rapides et facilement exploitables, des caractéristiques d'une crue correspondant à un risque donné. S'il est insuffisant de se contenter de leurs résultats seuls, ces

(*) Présentement au LCPC Nantes. méthodes sommaires sont un outil irremplaçable de transfert des connaissances acquises depuis des stations voisines vers un site.

En effet, de récents travaux en cours [15], démontrent que les caractéristiques de crues nécessaires et utiles pour aborder avec un maximum d'objectivité les inondations et leurs impacts, c'est-à-dire les courbes débits-duréesfréquences, peuvent être transférées d'une station à un site avec d'autant plus d'efficacité qu'elles sont normées avec des caractéristiques représentatives des crues. Parmi ces dernières, les caractéristiques dites $Q I X A_{10}$ et $D$ (voir le tableau des notations utilisées) sont parmi les plus efficaces. Les méthodes susceptibles d'estimer ces paramètres sont donc appréciées.

De telles méthodes existent, mais fournissent des résultats relativement peu précis. Elles peuvent gagner un peu en précision si l'on tente de les régionaliser.

Par ailleurs, les problèmes sont particulièrement aigus pour les petits bassins amont torrentiels où les données hydrométriques sont rarissimes.

\section{Mapping of regional knowledge in observable floods. South-East of France (Alps)}

In the framework of a several-year and regional-cooperating programme of Alpine Hydrology, a project has dealed with applied research on flood observations. More than 200 stations where available, with more than 20 basin characteristics and the results coming from a similar project (300 stations) on daily rainfalls. The tools of Regional Hydrological methods were used, and rough models were calibrated in the CRUPEDIX or SOCOSE spirit. Results were mapped whith some innovation (recovered pseudo-specific discharges), a great attention given to lacks and errors (both mapped), and a grid size chosen as to forbid local micro-relief or micro-climate interpretations. 
Un groupe de travail réunissant divers laboratoires (CEMAGREF : Antony, Grenoble, Lyon et Aix-enProvence; CNRS: LAMA; SRAE: Rhône-Alpes et Provence-Côte-d'Azur), et exploitant les données disponibles régionalement (SRAE, SN, EDF-DTG, CEMAGREF, ...) s'est donc réuni ces dernières années, dans le cadre d'une opération dite de RTM, pour tenter de produire une méthode sommaire, mais opérationnelle, d'estimation des débits instantanés de crues sur les petits bassins du Sud-Est de la France. Une tentative d'extrapolation vers les bassins torrentiels amont était envisagée.

Divers documents de travail, à caractère opérationnel, ont été établis (voir bibliographie). Ils sont numérotés de 1 à 7 et sont disponibles auprès des antennes des différents services et laboratoires concernés.

Il restait à élaborer une synthèse de ces travaux présentant de manière claire les formules sommaires proposées et leurs limites d'application.

L'objectif de lisibilité maximale a dirigé toute la rédaction de cette synthèse. Le détail des méthodes ne sera donc pas repris et l'on peut toujours, pour plus de précisions, se référer aux rapports cités en références.

Malgré tout, dans un souci de clarté, les grandes lignes seront reprises et résumées.

Pour répondre au même objectif, doublé d'une volonté d'inciter à une lecture critique des résultats, les cartes " zonées » sont souvent présentées en vis-à-vis des cartes "des valeurs" attribuées aux différents bassins versants, pour une variable donnée.

Les cartes établies dans cette synthèse, plus qu'une représentation précise de résultats, doivent avant tout être considérées comme une représentation synthétique commode et simplifiée des résultats obtenus. Le mode de représentation des cartes interpolées traduit cet objectif: maille volontairement carrée, zones laissées blanches, faible définition spatiale ("pas» de $7 \mathrm{~km}$ ), etc...

Les résultats énoncés ici, rappelons-le, ne sont pas définitifs mais susceptibles d'être révisés et améliorés si l'on dispose de données complémentaires. En particulier, le bon usage de ces méthodes d'estimation sommaires, plutôt que d'utiliser leurs résultats tels quels, consiste à les utiliser comme "outil de transfert" des résultats de quelque(s) station(s) proche(s) jugée(s) représentative(s) sur le site en question. Ces stations "représentatives" peuvent ne pas avoir été prises en compte ici, et peuvent servir à caler localement les méthodes d'estimation sommaires présentées ici (par exemple en recalculant la valeur d'un coefficient de correction $R$ ).

La représentation cartographique des facteurs de correction à appliquer aux différentes formules d'estimation présentées est, de plusieurs points de vue, très intéressante :

- ces cartes visualisent directement les zones géographiques où les méthodes sommaires présentées ont des performances similaires; ceci peut être une aide au transfert de résultats cité plus haut, et à l'amélioration des formules elles-mêmes ;

- ces cartes permettent en outre, dans l'attente d'une amélioration ultérieure des formules, d'appliquer celles-ci avec une meilleure connaissance de la marge d'erreur attendue (s'il n'est pas possible de recaler la formule localement).

Les formules d'estimation proposées ici sont donc sommaires et entachées d'erreur, mais celle-ci est quantifiable de par la démarche statistique employée. Les formules sont donc à utiliser avec discernement, en tenant compte des intervalles de confiance présentés et des cartes des facteurs de correction régionaux et, si possible, en commun avec d'autres méthodes d'estimation. Enfin, l'erreur sur le résultat doit être indiquée lors d'une utilisation pratique.

Cet article résume un rapport de synthèse complet publié sous l'égide du Programme « Risques naturels en montagne ", thème " Crues ", piloté sous IX ${ }^{e}$ Plan, contrat EtatRégion Rhône-Alpes [17].

\section{Présentation générale}

\subsection{La spécificité géographique de la région Sud-Est}

Il s'agit d'une région très contrastée, où l'on trouve aussi bien le bord de mer de la Côte d'Azur, que le toit de l'Europe au sommet du Mont Blanc à $4807 \mathrm{~m}$.

Chaque relief, chaque type d'exposition au soleil, ou la proximité de la mer, entraînent des nuances importantes du climat régional, et donc du régime des crues.

\section{1) L'extrême diversité des régimes des cours d'eau}

Dans la région Sud-Est, tous les types de régimes se trouvent représentés :

glaciaire, glacio-nival, nivo-glaciaire, nival, nivo-pluvial, pluvio-nival, pluvial.

On peut néanmoins distinguer deux contextes différents :

Au Nord : les pluies sont grossièrement équi-réparties sur les 12 mois de l'année. La température, l'ensoleillement et l'évapo-transpiration constituent donc les facteurs principaux d'influence climatique sur le régime des débits.

Les crues sont abondantes en volume au printemps, en liaison avec la fonte des neiges. Par contre, les débits de pointes (instantanés) les plus importants sont surtout observés en Automne, liés aux pluies extrêmes.

$A$ u Sud: les fortes variations saisonnières des pluies Cévenoles et méditerranéennes typent nettement les régimes des débits, en plus des effets dus à l'évaporation. Les débits, en volume et en pointe, présentent des maximums très marqués en Automne.

\section{2) Les climats}

Toutes les études « régionales » ([3], [1], [2], etc...) démontrent la prédominance des facteurs climatiques dans l'explication, par une approche statistique, des débits de crues extrêmes (et notamment du débit instantané de crue décennal).

Les météorologues proposent des découpages en "climats régionaux $"$ : le principe du zonage climatique régio- 
nal [4] repose sur le regroupement des stations d'observation ayant des valeurs proches pour les moyennes saisonnières ou annuelles des principales variables climatiques retenues. La zone déterminée est alors supposée homogène du point de vue climatique.

Ces climats régionaux sont surtout valables en plaines et pourtours montagneux, où la distance caractéristique associée est de l'ordre de $100 \mathrm{~km}$.

En montagne, le climat régional n'existe plus.

On peut, plus difficilement, définir un climat subrégional en raisonnant à une échelle de l'ordre de $30 \mathrm{~km}$ et en s'appuyant sur le régime pluviométrique.

Le CNRS a produit une carte climatique détaillée de la France au $1: 250000[16]$.

Mais surtout, il faut introduire la notion de topoclimat (caractérisé par une échelle de l'ordre de $10 \mathrm{~km}$ en plaine, et de l'ordre de $1 \mathrm{~km}$ en montagne), qui correspond à des facteurs d'influence du second ordre engendrés par la topographie environnant le site. On peut citer les phénomènes météorologiques suivants :

- l'opposition classique de l'exposition au soleil, initiatrice de phénomènes thermiques très importants en montagne entre l'adret, le versant exposé au Sud, et l'ubac, le versant exposé au Nord ;

- les brises de pentes et les brises de vallée, dues à des contrastes de température :

les phénomènes de brises sont dépendants des conditions météorologiques générales (inversion thermique due par exemple à des conditions anticycloniques, ensoleillement, etc...) et varient en cours de journée (vitesse et direction du vent);

- l'effet de foehn qui se produit quand un vent plus ou moins perpendiculaire à une crête franchit celle-ci :

en amont du relief, l'air s'élève et se refroidit jusquà ce que la vapeur d'eau se condense avec restitution de chaleur latente, ce qui réduit la décroissance de température suivant l'altitude. Ces nuages peuvent donner lieu à des précipitations localisées.

En aval du relief, l'air désaturé s'abaisse, se réchauffe encore davantage, et la couverture nuageuse peut disparaître. Ce versant, sur une zone de quelques $\mathrm{km}$ en aval de la crête, bénéficie globalement d'une augmentation de la température par rapport à l'autre versant, d'un meilleur ensoleillement, d'une plus faible pluviométrie (ceci si le phénomène se répète régulièrement).

Cet effet de foehn peut accélérer la fonte du manteau neigeux sur certaines pentes, en hiver ou au printemps.

\section{3) Le type de sol et la végétation}

Ils peuvent être à l'origine de micros-climats à une échelle de l'ordre de la centaine de mètres.

Ils peuvent influencer profondément l'écoulement de l'eau, à condition de ne pas être saturés, ce qui est rapidement le cas lors de pluies très intenses ou répétées sur une courte période de temps.

Dans les études qui ont précédé l'élaboration de cette synthèse, quelques variables représentatives du sol et de la couverture végétale ont été testées statistiquement (méthode de type CRUPEDIX), mais n'ont pas permis d'apporter une explication supplémentaire significative, au sens statistique, des débits instantanés maximaux décennaux ou biennaux [8].

Pour plus de renseignements sur le sol, le sous-sol et la végétation de la région Sud-Est, le lecteur peut se référer aux travaux suivants:

- le CNRS a édité des cartes de la végétation de l'ensemble de la France au 1:1250000. On pourra aussi y trouver des renseignements sur la nature du sol;

- pour la géologie, on peut consulter la « Synthèse géologique du Sud-Est de la France" (Orléans, BRGM 1984).

\section{4) L'action de l'homme}

Celle-ci est très importante dans la région Sud-Est et vient modifier constamment les conditions naturelles du milieu. On peut citer, à titre d'exemples : les multiples barrages et retenues sur les cours d'eau; l'aménagement urbain dont l'impact sur l'écoulement des eaux, ou sur ses conséquences, est souvent mal maîtrisé (l'inondation de Nîmes en octobre 1988 en est un exemple); les stations de sport d'hiver en montagne, où les pistes de ski sont un facteur de ruissellement important, etc...

Tous les bassins versants présentés dans cette synthèse sont à influence artificielle supposée négligeable.

\subsection{Présentation de la synthèse régionale}

\subsubsection{Variantes de CRUPEDIX}

En s'inspirant de la méthode CRUPEDIX développée dans la synthèse nationale [1], une analyse multivariable a été entreprise sur l'ensemble de la région Sud-Est afin de tester régionalement, et éventuellement d'affiner, les résultats nationaux.

Les étapes de cette étude sont développées dans les différents rapports intermédiaires ([7] à [12]).

Il a d'abord fallu effectuer un choix des petits bassins versants à partir de deux exigences contradictoires : petite taille, d'une part, nombre suffisant pour effectuer une analyse statistique, d'autre part.

Selon les analyses successives ce nombre a varié pour tenir compte des données disponibles, de 80 à 235 .

Il a ensuite fallu rassembler toutes les données susceptibles d'expliquer la variance du débit instantané maximal décennal $\left(Q I X A_{10}\right)$, ou biennal $\left(Q I X A_{2}\right)$. Les données reprises dans cette synthèse sont exposées dans le chapitre suivant.

En limitant le nombre des bassins versants dans certains échantillons, on a pu faire l'effort de tester des variables explicatives nouvelles, dont l'obtention, pour certaines d'entre elles, est longue et fastidieuse (cf. annexe et [8]).

Finalement, par rapport à la formule nationale, on n'a pu introduire qu'une variable nouvelle dans les dernières formules d'estimations: il s'agit du module interannuel divisé par la surface du bassin versant considéré $Q A / S$ (en $\mathrm{m}^{3} / \mathrm{s} . \mathrm{km}^{2}$ ), c'est-à-dire du module dit spécifique. 
Le travail de synthèse de ces travaux, qui ont porté sur plusieurs années, comprend l'édition de cartes en couleurs très lisibles.

Certaines de ces cartes représentent des zones correspondant à des limites de classes de valeurs :

Les cartes d'indice de crues: uniquement indicatives, elles doivent aussi conduire à une meilleure prise de conscience des problèmes liés à la surface d'étude lors des tentatives d'estimation de paramètres débimétriques à partir de formules prédéterminées.

Les cartes des facteurs de correction à appliquer aux formules d'estimations: elles représentent une interpolation, à partir de données partielles, de données qui sont le résultat d'une formule d'estimation résultant d'une étude statistique.

De plus, la surface des bassins versants n'est pas prise en compte lors de l'interpolation spatiale : chaque valeur est ramenée au centre de gravité du bassin considéré.

Ces cartes doivent donc servir à une utilisation critique des formules proposées dans cette synthèse et non à une utilisation automatique.

Malgré ces réserves, on voit nettement apparaître des " zones ", ou " sous-régions ", où les formules, soit surestiment, soit sous-estiment, soit encadrent relativement bien, le débit de crue recherché. Cela démontre une cohérence dans la variance, qu'il reste à expliquer, du débit instantané de crue, qu'il soit décennal ou biennal.

Mais attention: les informations manquent totalement sur la variabilité locale liée au relief (altitude, exposition, pente) :

le zonage présenté, significatif à l'échelle des cartes éditées (et compte tenu de la faible définition des images), ne tient pas compte des effets locaux du relief à une échelle plus grande, de " pas » inférieur à quelques dizaines de km. Ces effets restent à estimer ultérieurement.

Il est donc conseillé, lorsque cela est possible, de recaler localement la valeur du coefficient de correction régional à partir de station(s) de référence voisine(s).

\subsubsection{Régionalisation de SOCOSE}

Une tentative de régionalisation de la méthode SOCOSE nationale, d'estimation du débit instantané maximal décennal $Q I X A_{10}$, et de la période caractéristique de crue de fréquence décennale $D$, a aussi été tentée sur 62 bassins versants. Les résultats sont médiocres.

Les formules nationales n'ont pas pu être améliorées et on a procédé à un calage régional "à postériori " de la formule d'estimation de l'interception potentielle $J$.

\section{Présentation des données utilisées dans les métho- des CRUPEDIX et CRUPEDEUX}

\subsection{Le choix des stations hydrométriques}

La période d'observation à la station doit être supérieure à 7 années. Les bassins versants sont non karstiques, ou alors à bassin versant connu et dont la surface est bien déterminée. Les influences artificielles ont été supposées négligeables pour les forts débits.

Les localisations sommaires des stations sont indiquées sur plusieurs des cartes "à point» (non interpolées) présentées ici. Les noms de stations, noms de cours d'eau, et codes INSEE sont détaillés dans les rapports intermédiaires ([7] et [8], surtout), ainsi que des localisations détaillées des bassins et stations.

$\mathrm{Au}$ total, 235 stations ont été retenues, réparties sur toute la région Sud-Est.

\subsection{Les bassins versants associés aux stations hydrométri- ques}

Un total de 121 bassins ont une surface inférieure ou égale à $100 \mathrm{~km}^{2} ; 7$ seulement ont une surface supérieure à $1000 \mathrm{~km}^{2}$; la médiane est égale à $90 \mathrm{~km}^{2}$.

L'histogramme de la figure 1 représente la répartition en effectifs de la surface des 235 bassins analysés:

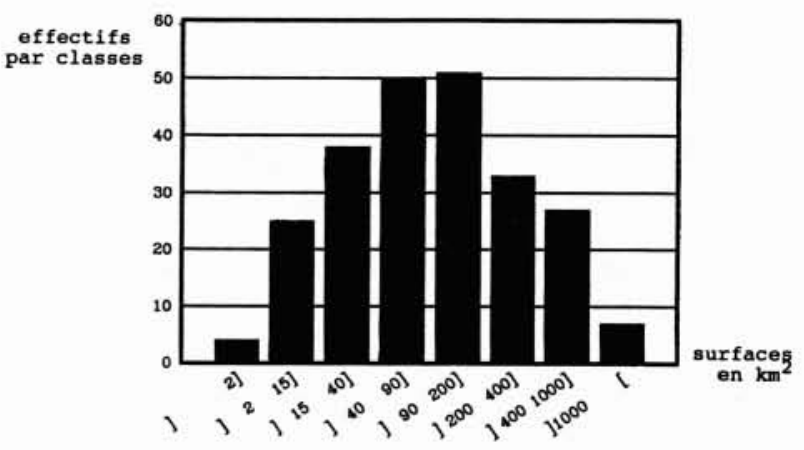

1. Répartition statistique des surfaces analysées.

\subsection{La pluie journalière maximale décennale $\left(P J X A_{10}\right)$, ou biennale $\left(P J X A_{2}\right)$, en $\mathrm{mm}$}

Il s'agit de la valeur de la pluie locale décennale, ou biennale, d'une station pluviométrique jugée représentative du bassin versant.

Les géographes du LAMA ont exploité leurs connaissances sur l'hydrologie du secteur pour choisir ces stations pluviométriques représentatives : ce ne sont pas toujours les plus proches des bassins versants.

Ces données viennent principalement des résultats de l'analyse des fortes pluies de 1 à 10 jours menée sur 300 postes pluviométriques du Sud-Est de la France [5].

Les données avaient été récoltées au sein du fichier pluviométrique du Bureau de l'Eau de la Direction de la Météorologie nationale et répondent aux critères suivants : - période d'observation de plus de 17 années exploitables,

- peu ou pas d'observations isolées manquantes. 
Ce sont des pluies locales centrées sur les intensités maximales et supérieures à un seuil. Ce seuil est fixé à $16 \mathrm{~mm}$ dans la phase d'échantillonnage.

Les valeurs décennale et biennale utilisées sont les quantiles correspondants, déterminés à partir d'un ajustement d'une loi de Montana généralisée, sur les plus fortes valeurs retenues: le seuil initial d'échantillonnage est donc toujours dépassé dans la phase d'ajustement de la loi statistique.

Le document [17] contient une cartographie interpolée sommaire de $P J X A_{10}$.

\subsection{Le débit instantané maximal décennal $\left(Q I X A_{10}\right)$, ou biennal $\left(\mathrm{QIXA}_{2}\right)$, «observé ", en $\mathrm{m}^{3} / \mathrm{s}$}

Il s'agit de la valeur, à la station hydrométrique, du débit instantané maximal annuel de période de retour 10 ans ou 2 ans. Ces valeurs sont déterminées à partir d'une loi de Gumbel ajustee sur les valeurs des deux plus fortes crues mensuelles de chaque année d'observation soit, pour $\mathrm{n}$ années d'observation, $2 n$ valeurs de $Q I X M$ (débit instantané maximal mensuel).

Origine des données: banque de données SRAE (ARHMA-HYDRO), Service de la Navigation (cellule d'étude des débits de Grenoble), DTG de l'EDF, CEMAGREF d'Aix-en-Provence, d'Aix-les-Bains, de Grenoble puis Lyon.

\subsection{Le module interannuel $(Q A)$ en $\mathrm{m}^{3} / \mathrm{s}$}

Il est obtenu en faisant la moyenne des débits annuels pour la période d'observation.

Il est connu pour 216 des 235 stations hydrométriques retenues.

Cette variable a été introduite car :

- elle est assez significative statistiquement ;

- elle est connue, par nécessité, quelque soit la méthode employée, chaque fois que les crues sont étudiées sur un site, lui-même étudié pour des motifs de "ressources en eau " dans lesquels la connaissance du module interannuel est incontournable : ouvrages de prise en rivière, pompages, dérivations, barrages, etc... ;

- elle peut être estimée à partir de formules simples à utiliser [6].

\section{Présentation du traitement des données par les méthodes de type CRUPEDIX et CRUPEDEUX}

\subsection{L'analyse statistique multivariable}

Les variables introduites dans les programmes de calculs des corrélations couvrent un grand nombre de caractéristiques des bassins versants. On peut citer (cf. annexe et [8]) :
— des données morphométriques

- des données climatiques

— des données hydrauliques

- des données granulométriques

- des données de couverture en forêts, en lacs, en marais, en glaciers.

On a souvent utilisé le logarithme népérien des valeurs comme variable, et non les valeurs physiques directement ; entre autres : In $(S), \ln \left(Q I X A_{10}\right)$ ou $\ln \left(Q I X A_{2}\right), \ln (Q A / S)$, $\ln \left(P J X A_{10}\right)$ ou $\ln \left(P J X A_{2}\right)$.

Ceci permet d'avoir une variation plus linéaire, les unes par rapport aux autres, des variables introduites dans le programme, sans pour autant que ce soit le cas des variables physiques échantillonnées, et sans pour autant que leurs distributions marginales soit Normales stricto sensu.

De plus, dans une estimation de crue, l'erreur s'apprécie en valeur relative. Il est donc intéressant de remplacer la variable physique à estimer par son logarithme, ce qui donne le même écart, en valeur absolue, pour une valeur surestimée ou sous-estimée d'un même facteur multiplicateur ou diviseur.

Des tests de significativité, test $\mathrm{t}$ de Student, ont été réalisés sur les coefficients de corrélations partielles entre chacune de ces variables "explicatives" et la variable à expliquer, $Q I X A_{10}$ ou $Q I X A_{2}$. Ils ont permis de sélectionner les variables explicatives qui ont été retenues pour établir, par régression linéaire multiple, la formule d'estimation du débit instantané de crue recherché.

Au seuil $1 \%$, retenu dans toutes les études intermédiaires, le coefficient de corrélation partielle doit être supérieur à :

$$
\left[t_{1 \%}^{2} /\left(t_{1 \%}^{2}+n-p\right)\right]^{1 / 2}
$$

avec $n=$ nombre d'observations, $p=$ nombre de variables (explicatives + expliquée).

Pour un nombre de degré de liberté $(n-p)$ toujours proche de 200, la loi de Student est approximée par une loi Normale et $\mathrm{t}_{1} \%$ est pris égal à 2.58 .

Une fois la formule d'estimation établie, on trace le graphe de contrôle $\ln \left(Q I X A_{10}\right)=f\left\{\ln \left(Q I X A_{10}\right)\right\}$, ou $\ln \left(Q I X A_{2}\right)=f\left\{\ln \left(Q I X A_{2}\right)\right\}$, et les graphes de corrélation partielle entre chacune des variables explicatives, exprimées en logarithmes, et la variable à expliquer, $\ln \left(Q I X A_{10}\right)$ ou $\ln \left(Q I X A_{2}\right)$.

Ces graphes permettent de vérifier la linéarité et l'homoscédasticité des nuages de points, et permettent de déceler d'éventuels points marginaux "anormaux ». On vérifie ainsi la validité des coefficients de corrélation calculés par le programme.

On vérifie enfin que les variables explicatives retenues ont une "signification physique " et que l'exposant qui apparaît dans les formules correspond bien à ce que l'on s'attend à trouver :

par exemple :

$<1$ pour la surface du bassin versant, dû à l'effet de laminage de l'amont vers l'aval

$>1$ pour la pluie journalière maximale décennale ou biennale, le rendement hydrologique croissant plus vite que linéairement. 


\subsection{Le calcul et la cartographie des erreurs d'estimation}

A partir de chacune des formules retenues, on calcule ensuite, pour chaque bassin versant, le débit instantané maximal décennal, ou biennal, estimé par la formule, et le facteur d'erreur de l'estimation $r$ :

$$
Q I X A_{10} / Q I \hat{X} A_{10} \text { ou } Q I X A_{2} / Q I \hat{X} A_{2}
$$

Ce facteur, sans dimension, correspond :

- lorsqu'il est supérieur à 1 , à une sous-estimation du débit instantané maximal, décennal ou biennal, par la formule.

- lorsqu'il est inférieur à 1 , à une surestimation du débit instantané maximal, décennal ou biennal, par la formule.

Les valeurs de ce rapport ont été réparties en 5 classes :

$$
\begin{aligned}
\mathrm{r} & <0,50 \\
0,50<\mathrm{r} & <0,75 \\
0,75<\mathrm{r} & <1,33 \\
1,33<\mathrm{r} & <2,00 \\
2,00 & <\mathrm{r}
\end{aligned}
$$

A partir de ces classes, deux types de cartes en couleurs sont réalisées :

- une carte brute, à valeurs ponctuelles, où apparaît la classe à laquelle appartient la valeur du facteur d'erreur de l'estimation pour chaque bassin versant de l'étude. Ce facteur représente aussi le "facteur multiplicateur" qu'il faudrait appliquer à la formule, pour retrouver la valeur observée du débit à estimer ;

- une carte interpolée, et maillée, où est réalisé un zonage de la tendance moyenne des valeurs de ces facteurs sur l'ensemble de la région Sud-Est.

Cette carte peut être utilisée, avec prudence, pour corriger les résultats des formules d'estimation grâce à l'introduction d'un facteur régional de correction (voir §8).

La technique utilisée pour réaliser les cartes interpolées est décrite dans [17].

Les limites de zones sont volontairement laissées floues, non lissées, et sans dessin de lignes isovaleurs, car :

- ces limites ne sont pas à respecter dans le détail ;

- les cartes n'indiquent que la tendance moyenne, de la variable cartographiée, à l'intérieur de chaque zone.

Cette tendance n'est elle-même définie que par les seules données observées qui sont irrégulièrement réparties et dont la densité peut être faible dans certaines régions.

Pour cette raison, il a été défini un rayon maximal (Ray) de recherche pour l'interpolation, figuré par un cercle centré sur le point à interpoler. Si aucune donnée n'est trouvée à l'intérieur de ce cercle, l'interpolation n'a pas lieu, et la zone correspondante est laissée blanche sur la carte.

Ce rayon "Ray " varie en fonction de l'objectif de la carte : il est plus faible pour les cartes d'indices de crues, qui ne sont qu'indicatives, que pour les cartes de facteur de correction, qui peuvent servir à une meilleure application des formules, car nous considérons dans ce dernier cas qu'il vaut mieux donner l'information, même avec des réserves, plutôt que de ne rien donner du tout. Rappelons que la meilleure utilisation de ce facteur de correction, consiste à procéder à un calage régional de sa valeur à partir de stations voisines du site étudié.

Enfin, dans toutes les cartes qui ont été élaborées, les valeurs des différentes variables cartographiées sont reportées au centre de gravité de chaque bassin versant, bassins versants qu'il a fallu identifier à partir des cartes IGN au $1 / 200000$, et non pas à l'emplacement géographique des stations de mesures débimétriques, ce qui était le cas précédemment.

La différence peut être importante pour les bassins spatialement étirés, ou de grande superficie.

Cette manière de faire est plus adéquate pour des valeurs jugées représentatives des bassins versants mais elle doit prendre en compte les nuances suivantes:

- la valeur réelle au centre du bassin versant est rarement celle attribuée à ce bassin dans son ensemble (cela d'autant plus que le bassin est plus grand);

- la variation des valeurs rencontrées à l'intérieur d'un même bassin versant n'est pas représentée. Elle peut être importante pour des bassins de surface importante et/ou situés en zone montagneuse.

Malgré toutes ces réserves, la validité des cartes établies par la cartographie automatique, qui ne tient compte ni du relief, ni de la taille spécifique de chaque bassin versant, a été confortée en comparant le résultat obtenu pour certaines variables, aux cartes interpolées des mêmes variables dessinées manuellement à partir des mêmes données et avant toute cartographie automatique : les résultats sont similaires.

\section{Les indices de crues décennales}

\subsection{L'indice de débit de crue décennal pseudospécifique}

Pour chaque bassin versant, à partir de la seule connaissance du débit instantané maximal décennal $\left(Q I X A_{10}\right)$, et de la surface $(S)$ du bassin, on peut calculer un indice de crue :

le débit instantané maximal décennal pseudospécifique $Q I X A_{10} / S^{0,8}$ exprimé en $\mathrm{m}^{3} / \mathrm{s} \cdot \mathrm{km}^{1,6}$.

Cet indice de crues a été cartographié pour toute la région Sud-Est à partir des valeurs calculées sur les 235 bassins versants de l'étude (carte 4-1a de la figure 2, page 357). Une carte interpolée existe dans [17].

\subsection{L'estimation de débits instantanés décennaux spécifi- ques pour une aire de référence donnée: comparaison des effets de surface}

L'importance des effets d'échelle spatiale en hydrologie, et la différence fondamentale qui existe entre débits spécifiques et débits pseudo-spécifiques est à l'origine de ce $\S 42$. 
On ne peut pas dresser directement de carte de débits spécifiques en calculant les rapports $Q I X A_{10} / S$ car on obtiendrait des débits spécifiques valables pour des gammes de surface différentes (de 0,6 à $2510 \mathrm{~km}^{2}$ ). Or, un débit spécifique n'est pas le même pour n'importe quelle surface prise en compte.

Par contre, on peut calculer, à partir du débit instantané décennal pseudospécifique, pour chaque bassin versant, le débit instantané de crue décennale pour une surface donnée (exprimé en $\mathrm{m}^{3} / \mathrm{s}$ ). C'est ce que l'on a fait pour des surfaces de 1 hectare, $1 \mathrm{~km}^{2}$, et $100 \mathrm{~km}^{2}$.

Pour pouvoir comparer les résultats, on a divisé les trois types de débits obtenus par la surface prise en compte soit $0,01 \mathrm{~km}^{2}, 1 \mathrm{~km}^{2}, 100 \mathrm{~km}^{2}$. On obtient ainsi des débits instantanés de crues décennales spécifiques, exprimés en $\mathrm{m}^{3} / \mathrm{s} \cdot \mathrm{km}^{2}$, calculés pour trois surfaces différentes, et que l'on peut comparer directement entre eux. La procédure suivie est résumée dans le tableau $n^{\circ} l$ et a conduit aux cartes 4-2a, 4-2b et 4-2c de la figure 2 : des " débits instantanés de crues décennales spécifiques " calculés pour une surface de $100 \mathrm{~km}^{2}$, une surface de $1 \mathrm{~km}^{2}$, et une surface de $0,01 \mathrm{~km}^{2}$.

Les valeurs indiquées par ces cartes sont uniquement indicatives d'une tendance moyenne, mais elles rendent bien compte de la hiérarchie des régions pour les fortes crues.

On constate, par ailleurs que les résultats sont très différents en fonction de l'aire de référence prise en compte pour le calcul de l'indice de crue spécifique.

Pour une surface de 1 hectare, le débit instantané de crue décennale par $\mathrm{km}^{2}$ est 2,5 fois plus important que pour une surface de $1 \mathrm{~km}^{2}$ et 6,5 fois plus important que pour une surface de $100 \mathrm{~km}^{2}$.

Plus l'aire prise en compte est petite, plus l'écart type des débits spécifiques estimés est grand, pour les 235 bassins, et plus la valeur des débits de crue spécifiques peut être importante.

Un débit instantané de crue spécifique établi pour une gamme de surface ne doit donc pas être utilisé en dehors de cette gamme de surface.

Il vaut mieux utiliser le débit instantané de crue décennale pseudospécifique, qui tient compte de l'effet de surface, pour avoir une idée très sommaire du débit instantané de crue décennale d'un bassin versant de surface quelconque.

\section{Remarques}

a) la médiane des surfaces des bassins versants étudiés dans cette synthèse est de $90 \mathrm{~km}^{2}$

Les valeurs des débits instantanés spécifiques de crues décennales calculées pour des bassins de $100 \mathrm{~km}^{2}$ sont donc bien centrées sur les observations (cf. $\S 22$ ).

Par contre, les valeurs calculées pour 1 hectare sont très fortement extrapolées. Elles le sont déjà largement pour $1 \mathrm{~km}^{2}$. Ces valeurs sont donc uniquement indicatives de ce que peuvent être les débits de crues décennales pour de tels petits bassins amonts.
Tableau $n^{\circ} 1$ : Estimation des débits spécifiques. A partir du débit pseudospécifique estimé en un site, on calcule,
pour ce site, 3 dobits spécifiques pour 3 surfaces de bassins
supposés représentatifs de ce site:
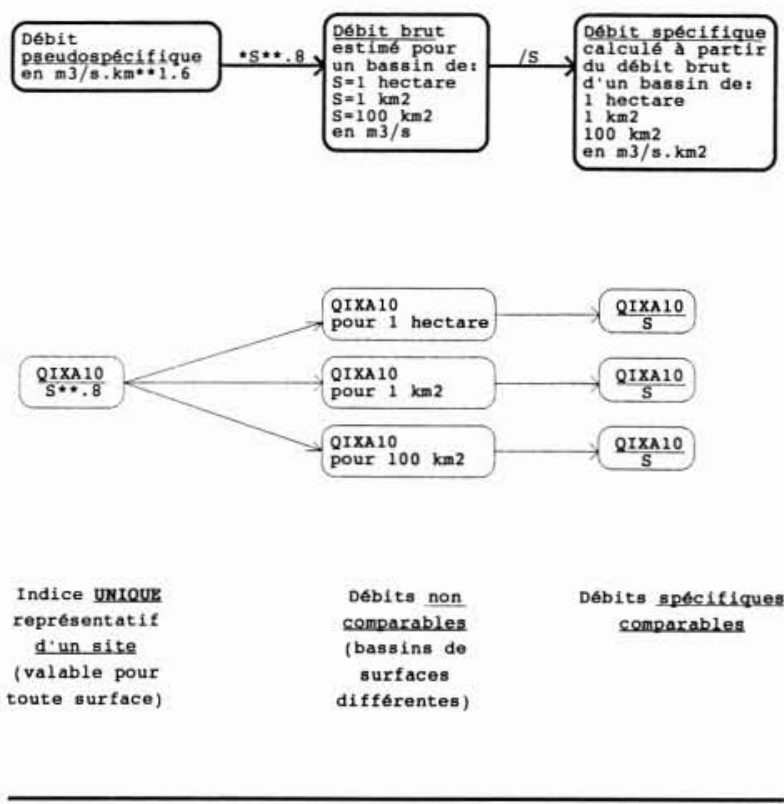

Des formules d'estimation mieux adaptées aux torrents devront être employées dès que possible.

Dans l'immédiat, les valeurs présentées, indicatives, témoignent de la tendance moyenne du phénomène "crue" dans le Sud-Est de la France, et permettent de différencier des régions «à risques » différenciées :

les valeurs calculées pour le débit instantané de crue décennale par $\mathrm{km}^{2}$, pour des bassins versants de 1 hectare, sont comprises entre $0,3 \mathrm{~m}^{3} / \mathrm{s} \cdot \mathrm{km}^{2}$ et $11,7 \mathrm{~m}^{3} / \mathrm{s} \cdot \mathrm{km}^{2}$, soit un facteur de variation de 1 à 40 ; une valeur atteint même $26,7 \mathrm{~m}^{3} / \mathrm{s} \cdot \mathrm{km}^{2}$ [la Rouvière à ROUVIERE], soit près de $100 \mathrm{~mm} / \mathrm{h} \cdot \mathrm{km}^{2}$ sur 1 ha.

b) Les résultats présentés dans ce chapitre sont uniquement liés :

- aux quantiles décennaux de débits "observés", c'està-dire obtenus sur des séries observées sur 235 stations hydrométriques ;

- à la variation de débit avec la surface, variation supposée, conformément aux méthodes de type CRUPEDIX, être de la forme $Q I X A_{10}=f\left(S^{0,8}\right)$.

Dans ce qui va suivre, on abordera l'estimation des débits instantanés maximaux de fréquence donnée, en un site non observé, donc avec l'introduction de variables disponibles sur de tels sites. 


\section{Les formules d'estimation du débit instantané décennal $\left(Q I X A_{10}\right)$ produites par une méthode de type CRUPEDIX régionale}

Deux formules ont été retenues:

\subsection{Sans la connaissance du module interannuel $Q A$}

$$
\begin{aligned}
\hat{Q} I X A_{10} & \left.=S^{0,8} \cdot\left(P J X A_{10}\right) / 73\right)^{1,5} \\
r & =0,86 \\
r^{2} & =0,74 \\
s_{1 \mathrm{n}} & =0,67
\end{aligned}
$$

(valeurs calculées sur 235 bassins versants) avec : $Q I X A_{10}$ en $\mathrm{m}^{3} / \mathrm{s}, S$ en $\mathrm{km}^{2}, P J X A_{10}$ en $\mathrm{mm}$.

\subsection{Avec la connaissance du module interannuel $Q A$}

$Q A$ peut être connu, par exemple si le site étudié a fait l'objet d'une étude des apports en eau (problèmes de ressources), ou si l'on dispose de vieilles chroniques, alors que l'on ne possède pas, ou plus, d'information sur les débits de pointes.

QA peut aussi être estimé à l'aide de formules simples [6]

$$
\begin{aligned}
& \hat{Q} I X A_{10}=S^{0,81} \cdot\left(P J X A_{10} / 28,5\right)^{1.56} \cdot(Q A / S)^{0,46} \\
r= & 0,91 \\
r^{2}= & 0,82 \\
s_{1 \mathrm{n}}= & 0,55
\end{aligned}
$$

(valeurs calculées sur 216 bassins versants) avec: $\hat{Q} I X A_{10}$ et $Q A$ en $\mathrm{m}^{3} / \mathrm{s}, S$ en $\mathrm{km}^{2}, P J X A_{10}$ en mm.

$r$ est le coefficient de corrélation entre $Q I X A_{10}$ observé et $\hat{Q} I X A_{10}$ estimé par la formule ;

$r^{2}$ représente la part expliquée par la formule de la variance de $Q I X A_{10}$ ( $74 \%$ ou $82 \%$ suivant la formule);

$s_{\text {ln }}$ est l'écart type de l'erreur résiduelle $E$ de la formule exprimée en logarithmes népériens :

$$
\ln \left(Q I X A_{10}\right)=\ln \left(\hat{Q} I X A_{10}\right)+E
$$

(rappel : la moyenne de l'erreur $E$ est nulle).

A partir de $s_{\ln }$ nous pouvons calculer, en faisant l'hypothèse de Normalité de l'erreur résiduelle, un intervalle de confiance probabilisé pour ces deux formules :

\begin{tabular}{|l|c|c|}
\hline \multirow{2}{*}{} & \multicolumn{2}{|c|}{ intervalle de confiance } \\
\cline { 2 - 3 } & à $70 \%$ & à $90 \%$ \\
\hline formule (2d) & {$[1 / 2 Q ; 2 Q]$} & {$[1 / 3 Q ; 3 Q]$} \\
\hline formule (4d) & {$[4 / 7 Q ; 7 / 4 Q]$} & {$[0,4 Q ; 2,5 Q]$} \\
\hline
\end{tabular}

Cela veut dire que l'on a $70 \%$ de chance pour que le débit $Q I X A_{10}$ réel appartienne à l'intervalle $[1 / 2 \hat{Q} ; 2$ Q $]$ pour la formule $(2 \mathrm{~d})$ et à l'intervalle $[4 / 7 \hat{Q} ; 7 / 4 \hat{Q}]$ pour la formule (4d).
Ces résultats sont meilleurs que ceux obtenus à partir de la formule nationale : avec la formule nationale on obtient, pour les 235 bassins versants étudiés ici

$$
r=0,85, \quad r^{2}=0,72, \quad s_{1 \mathrm{n}}=0,71 .
$$

\section{Remarque :}

La formule nationale, appliquée sur la région Sud-Est, donne donc de moins bons résultats que lorsqu'elle est appliquée aux 630 bassins versants de la synthèse nationale (où $r=0,95, r^{2}=0,90, s_{\mathrm{ln}}=0,4$ ).

Ceci est dû notamment à une plage de surfaces beaucoup plus large dans la synthèse nationale, où les grands bassins améliorent beaucoup la qualité de la régression (voir le graphe de contrôle $\ln \left(Q I X A_{10}=f\left\{\ln \left(\hat{Q} I X A_{10}\right)\right\}\right.$ dans la synthèse nationale).

\subsection{L'amélioration de ces formules d'estimation}

Sur l'ensemble des 235 bassins versants pris en compte dans cette synthèse, on a calculé le $\hat{Q} I X A_{10}$ estimé par les deux formules d'estimation présentées ci-dessus.

On a ensuite calculé le facteur d'erreur d'estimation $Q I X A_{10} / \hat{Q} I X A_{10}$ pour chaque bassin versant, et pour chacune des deux formules. On a réparti les facteurs d'erreurs en 5 classes :

les limites de classes définies sont: 1/2, 3/4, 4/3, 2 .

- $Q I X A_{10} / \hat{Q} I X A_{10}=1 / 2$ représente une surestimation de 2 pour 1 du débit instantané de crue décennale par rapport au débit observé ;

- $Q I X A_{10} / Q I X A_{10}=2$ représente une sous-estimation de la moitié du débit instantané de crue décennale par rapport au débit observé.

Ces facteurs d'erreurs d'estimations sont présentés, pour chaque bassin versant, sur les cartes $5-1$ a et $5-2 a$ des figures 3 et 4 .

A partir de ces cartes, il est possible de définir, pour un bassin versant donné, un coefficient régional de correction à appliquer lors de l'utilisation des formules d'estimation. Ces formules deviennent :

$$
\begin{aligned}
& \quad \hat{Q} I X A_{10}=S^{0,8} \cdot\left(P J X A_{10} / 73\right)^{1,5} \cdot R 23 d \\
& \hat{Q} I X A_{10}=S^{0,81} \cdot\left(P J X A_{10} / 28,5\right)^{1,56} \times \\
& \times(Q A / S)^{0,46} \cdot R 45 d
\end{aligned}
$$

Les coefficients régionaux, $R 23 d$ et $R 45 d$, à appliquer sont représentés sur les cartes $5-1 \mathrm{~b}$ et $5-2 \mathrm{~b}$ des figures 3 et 4 .

Les cartes du facteur de correction de l'estimation, $Q I X A_{10} / \hat{Q} I X A_{10}$, visualisent aussi la part de la variance de $Q I X A_{10}$ qu'il reste à expliquer. Elles peuvent être utilisées comme sources d'information dans la recherche de nouvelles variables explicatives. 
4-1a

INDICE dO DEBIT INSTANTANE DECENNAL débit pseudo-specifique, $01 \times A 10 / \mathrm{s} * *_{0.8}$, en $\mathrm{m} 3 / \mathrm{s} . \mathrm{km} * *_{1.6}$

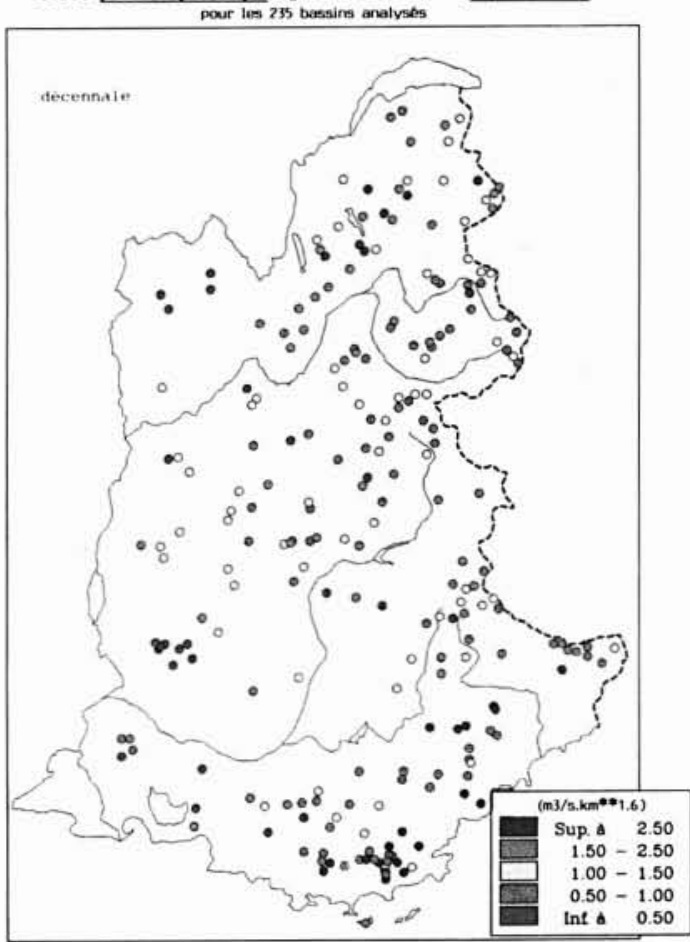

$4-2 b$

TENDANCE MOVENNE SUI BASSINS de $1 \mathrm{~km}$ ? du debit instantane décennal SPrCipiQuR, en $\mathrm{m} 3 / \mathrm{s}, \mathrm{km}$

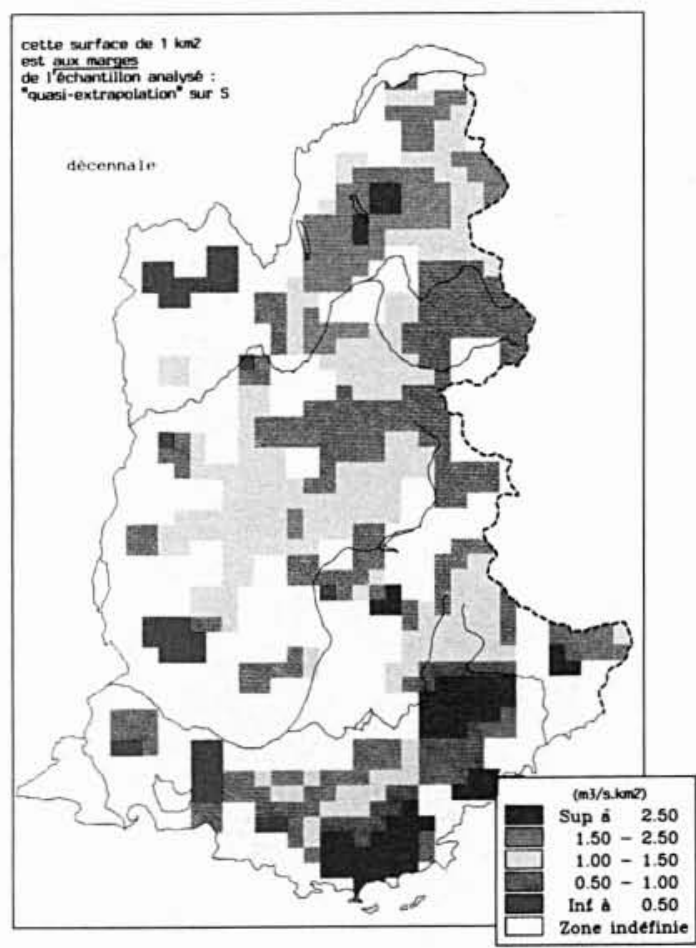

TENDANCE MOYENNE SUT BASSINS dO $100 \mathrm{kmz}$ du debit instantane decennal SPECIP1QUR, en $\mathrm{m} 3 / \mathrm{s} . \mathrm{km} 2$

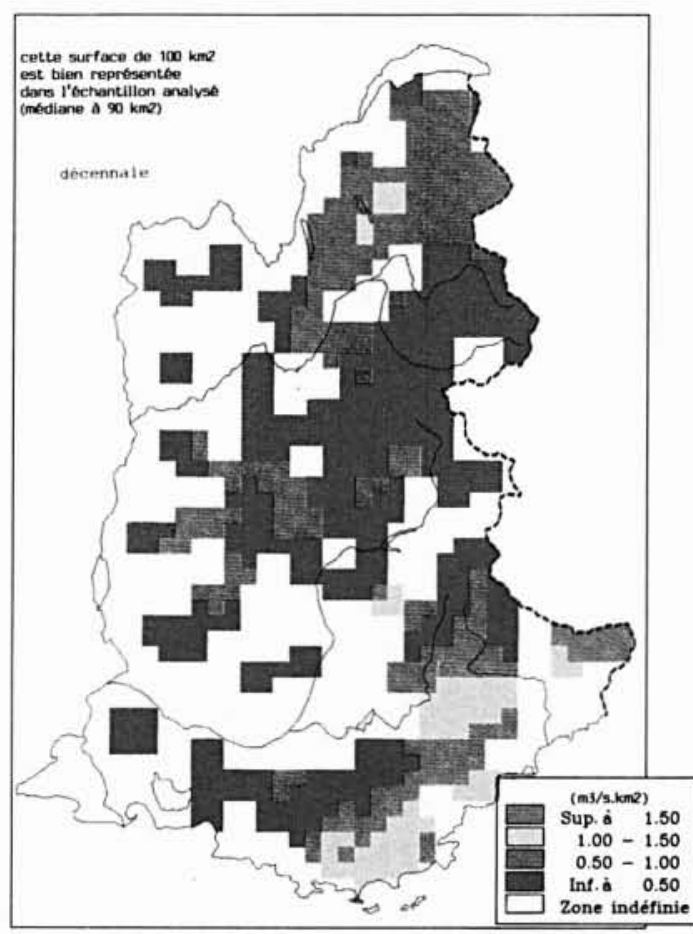

$4-2 c$

IENDANCF INDICATIVE BUF BASSINS de 1 he du debit instantane decennal SPECIFiQuR. en $\mathrm{m} 3 / \mathrm{s} \cdot \mathrm{km} 2$

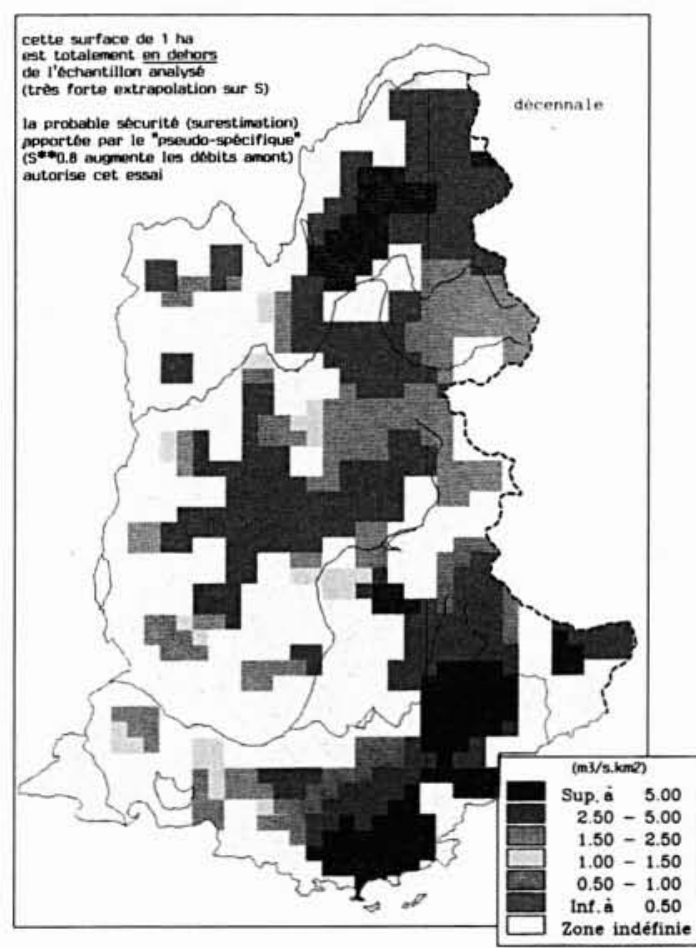

2. Effets d'échelles que permettent les débits pseudo-spécifiques. 
$3-1 \cdot a$

ANALYSE de I'ERREUR Q'ESTIMATION de $2 d$ rappel de la formule 2d, calée sur 235 bassins :

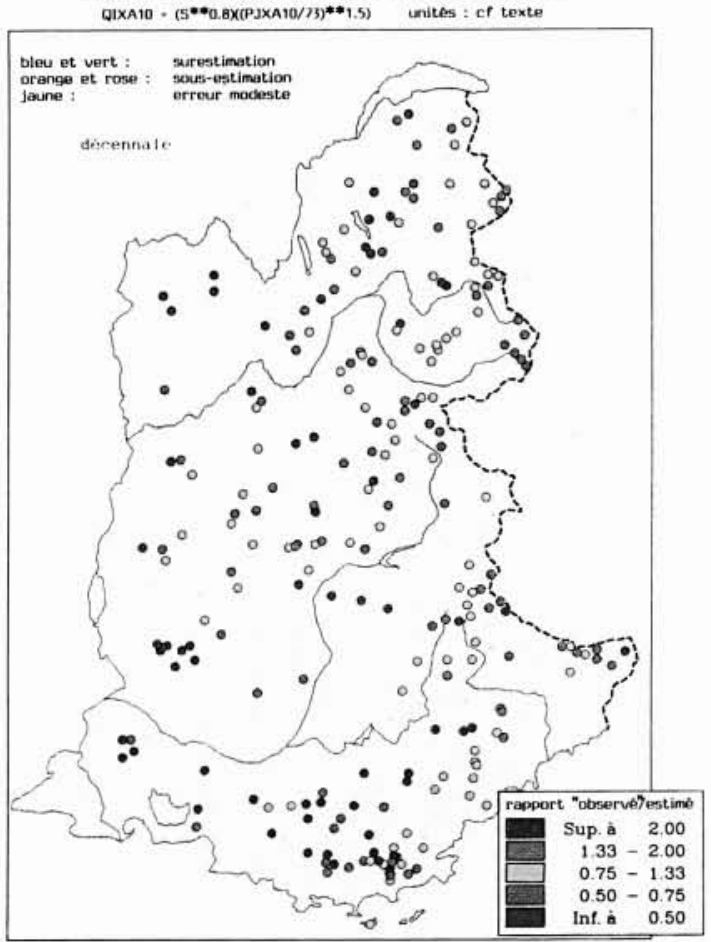

5-1b

IENDANCF MOYFNNF dU FACTEUR RZ3O R23d est le facteur de correction pour la formule 2d, qui devient alors 3d

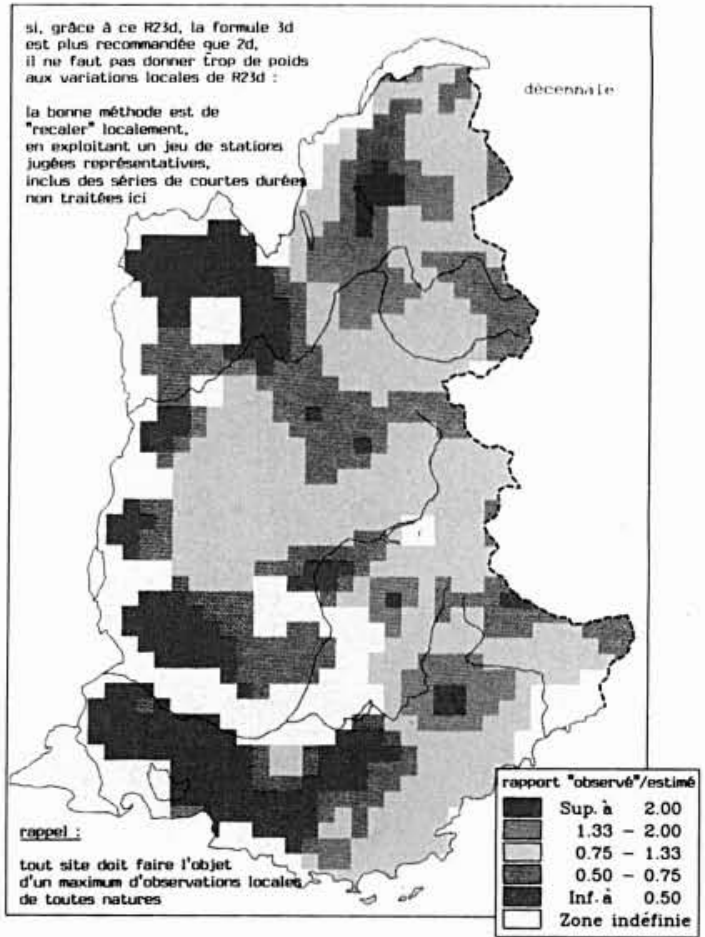

3. Incertitudes et coefficients de correction, pour la formulation CRUPEDIX (classique) régionalisée.

$b-2 a$

ANALYSE de I'ERREUR d'ESTIMATION de 4 d rappel de la formule $4 \mathrm{~d}$, calée sur 216 bassins :

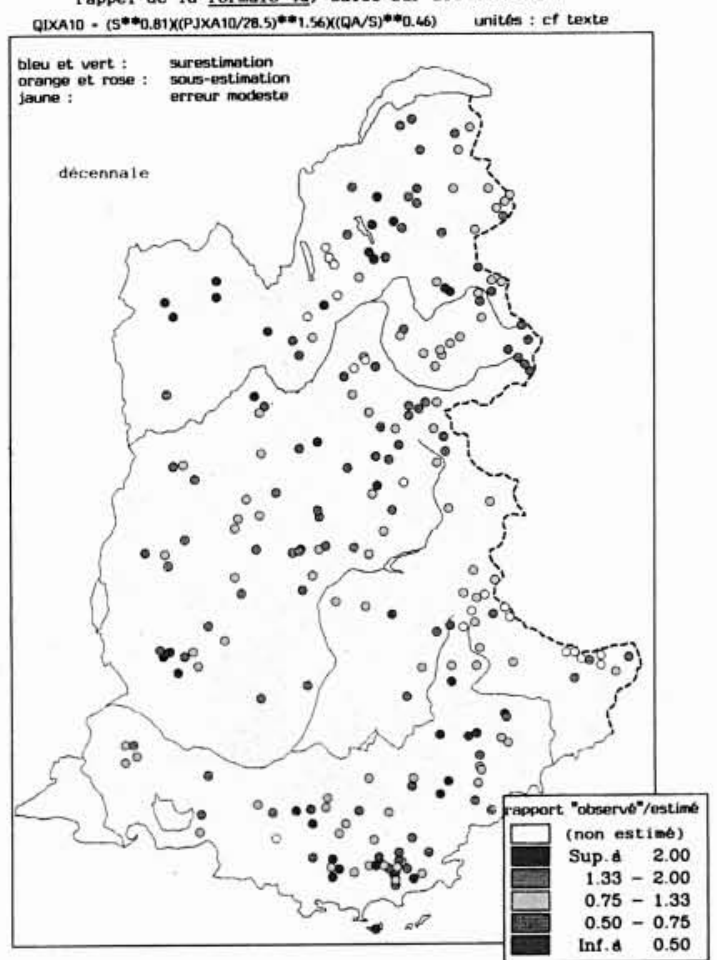

$5-2 b$

TENDANCE MOYENNE dU FACTEUR R45Q R45d est to facteur de correction pour ta formule 4d, qui devient alors $5 d$

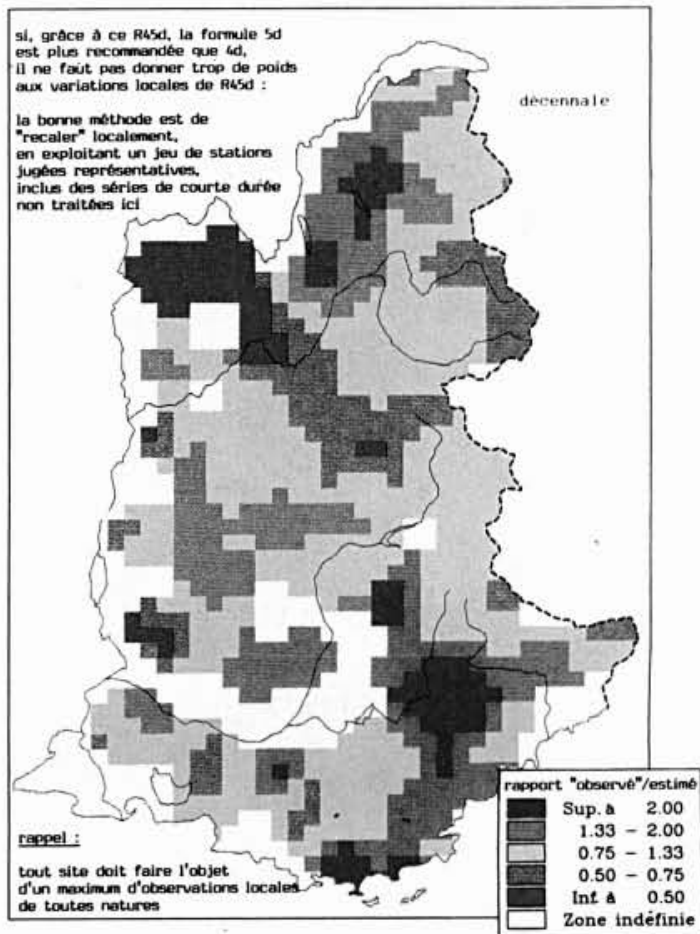

4. Incertitudes et coefficients de correction, pour la formulation " CRUPEDIX avec $Q A$ " régionalisée. 
$6-2 a$

ANALYSE de T'ERREUR A'ESTIMATION de 5b

rappel de la formule 5b. calee sur 189 bassins ?

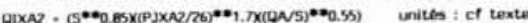

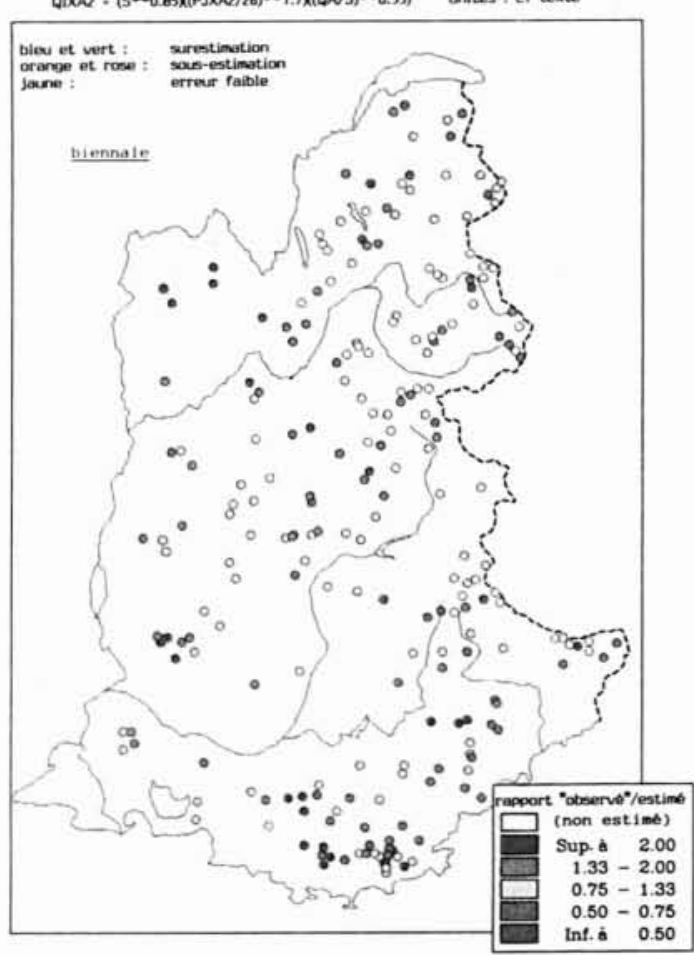

TENDANCE MQVENNE dU FACTEUR R56B

R56b est le facteur de correction pour la formule 5b. qui dovient alors 60

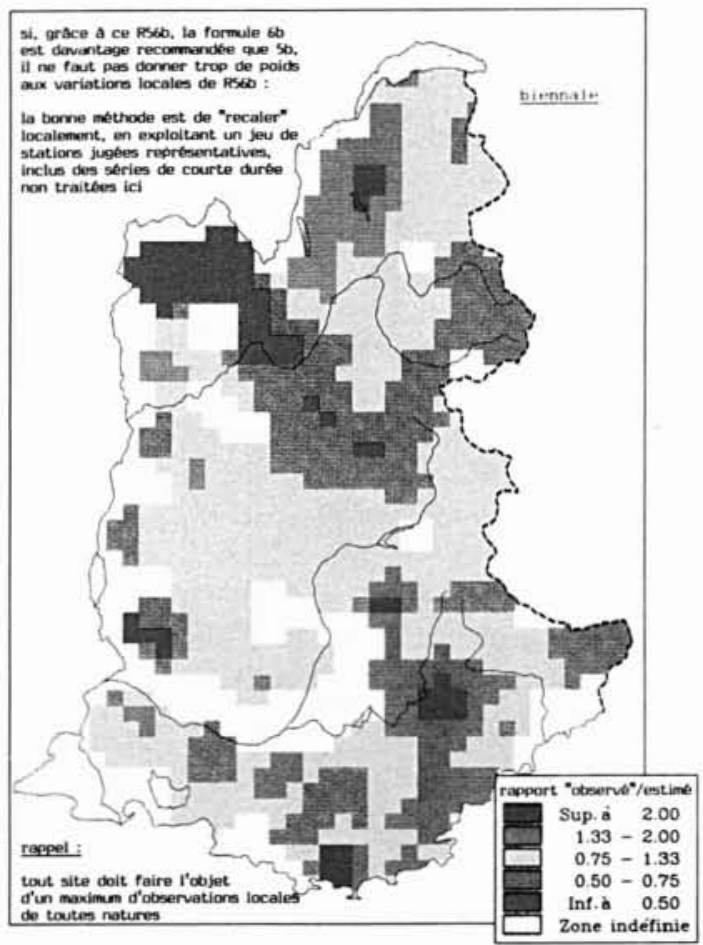

5. Incertitudes et coefficients de correction, pour la formulation “CRUPEDIX avec $Q A$ ” régionalisée.

6. Les formules d'estimation du débit instantané biennal $\left(Q I X A_{2}\right)$ produites par une méthode de type CRUPEDEUX régionale

Deux formules ont été retenues.

\subsection{Sans la connaissance du module interannuel $Q A$}

$$
\hat{Q} I X A_{2}=S^{0,8} \cdot\left(P J X A_{2} / 106\right)^{1,2}
$$

$r=0,87$

$r^{2}=0,76$

$s_{\mathrm{ln}}=0,68$

(valeurs calculées sur 208 bassins versants)

avec: $\hat{Q} I X A_{2}$ en $\mathrm{m}^{3} / \mathrm{s}, S$ en $\mathrm{km}^{2}, P J X A_{2}$ en $\mathrm{mm}$.

\subsection{Avec la connaissance du module interannuel QA [6]}

$$
\hat{Q} I X A_{2}=S^{0,85} \cdot\left(P J X A_{2} / 26\right)^{1,7} \cdot(Q A / S)^{0.55}
$$

$r=0,93$

$r^{2}=0,87$

$s_{\ln }=0,49$

(valeurs calculées sur 189 bassins versants) avec: $\hat{Q} I X A_{2}$ et $Q A$ en $\mathrm{m}^{3} / \mathrm{s}, S$ en $\mathrm{km}^{2}, P J X A_{2}$ en $\mathrm{mm}$.

$r$ est le coefficient de corrélation entre $Q I X A_{2}$ observé et $\hat{Q} I X A_{2}$ estimé par la formule.

$r^{2}$ représente la part expliquée par la formule de la variance de $Q I X A_{2}$ ( $76 \%$ ou $87 \%$ suivant la formule).

$s_{\ln }$ est l'écart type de l'erreur résiduelle $E$ de la formule exprimée en logarithmes népériens:

$$
\ln \left(Q I X A_{2}\right)=\ln \left(\hat{Q} I X A_{2}\right)+E
$$

(rappel: la moyenne de l'erreur $E$ est nulle).

A partir de $s_{\ln }$ nous pouvons calculer, en faisant l'hypothèse de Normalité de l'erreur résiduelle, un intervalle de confiance probabilisé pour ces deux formules:

\begin{tabular}{|l|c|c|}
\hline \multirow{2}{*}{} & \multicolumn{2}{|c|}{ intervalle de confiance } \\
\cline { 2 - 3 } & à $70 \%$ & à $90 \%$ \\
\hline formule (3b) & {$[+1 / 2 Q ; 2 Q]$} & {$[1 / 3 Q ; 3 Q]$} \\
\hline formule (5b) & {$[3 / 5 Q ; 5 / 3 Q]$} & {$[4 / 9 Q ; 9 / 4 Q]$} \\
\hline
\end{tabular}




\subsection{L'amélioration de ces formules d'estimation}

Sur l'ensemble des 208 bassins versants pris en compte, on a calculé le $\hat{Q} I X A_{2}$ estimé par les deux formules d'estimation présentées ci-dessus.

On a ensuite calculé le facteur d'erreur de l'estimation $Q I X A_{2} / \hat{Q} I X A_{2}$ pour chacun des bassins versants, et pour chacune des deux formules. Ces facteurs d'erreur ont été répartis en 5 classes :

les limites de classes définies sont: 1/2, 3/4, 4/3, 2 .

- $Q I X A_{2} / \hat{Q} I X A_{2}=1 / 2$ représente une surestimation de 2 pour 1 du débit instantané de crue biennale par rapport au débit observé ;

$-Q I X A_{2} / \hat{Q} I X A_{2}=2$ représente une sous-estimation de la moitié du débit instantané de crue biennale par rapport au débit observé.

Les facteurs d'erreurs d'estimations sont présentés, pour chaque bassin versant, sur la carte $6.2 \mathrm{a}$ de la figure 5 .

A partir de cette carte, il est possible de définir, comme pour les formules d'estimation des débits de crues décennales, un coefficient régional de correction, pour un bassin versant donné, à appliquer lors de l'utilisation des formules d'estimation. Ces formules deviennent :

$$
\hat{Q} I X A_{2}=S^{0,8} \cdot\left(P J X A_{2} / 106\right)^{1,2} \cdot R 34 b
$$

$\hat{Q} I X A_{2}=S^{0,85} \cdot\left(P J X A_{2} / 26\right)^{1.7} \cdot(Q A / S)^{0.55} \cdot R 56 b(6 \mathrm{~b})$

Les coefficients régionaux $R 56 b$ à appliquer sont représentés sur la carte 6-2b de la figure 5 (voir [17] pour la carte de $R 34 b$ ).

Les cartes du facteur de correction de l'estimation, $Q I X A_{2} / Q I X A_{2}$, visualisent aussi, comme pour $Q I X A_{10}$, la part de la variance de $Q I X A_{2}$ qu'il reste à expliquer. Elles peuvent être utilisées comme sources d'information dans la recherche de nouvelles variables explicatives.

\section{Remarque :}

Il est à noter que les cartes des facteurs de correction pour les formules décennales et pour les formules biennales présentent le même type de structure sous-jacente.

Ceci n'est pas totalement étonnant :

- on a calculé la corrélation entre les 2 variables $Q I X A_{10}$ et $Q I X A_{2}$ sur les 208 bassins où l'on connaissait le débit biennal : $Q I X A_{10}$ et $Q I X A_{2}$ sont liés à $98 \%$;

- on a calculé la corrélation entre les 2 variables $P J X A_{10}$ et $P J X A_{2}$ sur les 208 bassins où l'on connaissait la pluie biennale : $P J X A_{10}$ et $P J X A_{2}$ sont liés à $94 \%$;

- les valeurs de $Q I X A_{10}$ et $Q I X A_{2}$ " observés " sont deux quantiles de la même loi de Gumbel;

- les valeurs de $P J X A_{10}$ et $P J X A_{2}$ " observés " sont deux quantiles de la même loi de Montana.

\section{Essai de régionalisation de la méthode SOCOSE nationale}

Les formules nationales [1] ont été appliquées pour chacun des 235 bassins versants de l'étude. On a ensuite déterminé l'équation de régression linéaire entre débit « observé » et débit " estimé " :

$$
\ln \left(Q I X A_{10}\right)=1,14 \ln \left(\hat{Q} I X A_{10}\right)-0,556
$$

avec

$r=0,851$

$R^{2}=0,72$

$s_{\ln }=0,7$

On obtient donc, pour l'estimation du débit instantané maximal décennal à partir de la méthode SOCOSE nationale appliquée à la région Sud-Est, un intervalle de confiance à $70 \%$ de $\left[1 / 2 Q I X A_{10} ; 2 Q I X A_{10}\right]$, ce qui est comparable au résultat de la méthode à l'échelle nationale. Ce résultat n'est pas meilleur que celui obtenu par la méthode CRUPEDIX qui est beaucoup plus simple à mettre en œuvre.

Comme pour les méthodes CRUPEDIX et CRUPEDEUX, on a cartographié, au centre des 235 bassins analysés, les facteurs d'erreurs $\left(Q I X A_{10}\right.$ observé) $/\left(\hat{Q} I X A_{10}\right.$ calculé par SOCOSE nationale) :

- lorsque ce rapport est supérieur à 1, la méthode «SOCOSE nationale » sous-estime le débit instantané de crue décennal ;

- lorsque ce rapport est inférieur à 1, la méthode "SOCOSE nationale" surestime le débit instantané de crue décennal.

Un zonage de ces valeurs est présenté en [17]. On y remarque le même type de structure sous-jacente, toujours inexpliquée, que celui rencontré dans les cartes des facteurs de correction des méthodes CRUPEDIX et CRUPEDEUX (cartes 5-1b, 5-2b, 6-2b).

La mauvaise performance de la méthode SOCOSE étant due essentiellement à la mauvaise estimation, à partir des caractéristiques des bassins versants, de la durée caractéristique de crue $D$ et de l'interception potentielle $J$, on a tenté d'améliorer celles-ci.

\subsection{Essai d'amélioration de l'estimation de la durée carac- téristique de crue $D$}

Parmi les 235 bassins versants retenus, on a sélectionné 62 bassins pour lesquels on a déterminé, à partir des observations, la durée caractéristique de crue $D$.

On a ensuite procédé à des corrélations simples entre $D$ " observé » et $\hat{D}$ estimé par des formulations nouvelles » de SOCOSE régionalisée :

$$
\ln \left(D_{\text {observè }}\right)=a \ln \left(\hat{D}_{\text {estimè }}\right)+b .
$$

Les résultats fournis par les formules nationales n'ont pas pu être améliorés : $r=0,58, s_{\mathrm{ln}}=0,61$.

\subsection{Essai d'amélioration de l'estimation de l'interception potentielle $J$}

A partir de la cartographie des facteurs d'erreurs d'estimation $\left(Q I X A_{10}\right.$ observé $) /\left(Q \hat{Q} I X A_{10}\right.$ estimé) de la formule "SOCOSE nationale », on a pu définir des « zones », pour lesquelles on peut appliquer la formule avec un coefficient multiplicatif de correction différent de 1 .

Ces coefficients ont servi à modifier la valeur de la constante dans l'expression de $J$ nationale : 
$J=\mathrm{Cte}+21 \ln (S / L)-54[P A / P]^{0.5}, \quad$ où Cte $=260$.

$\mathrm{Si}$ la valeur moyenne de la constante reste bien égale à 260 sur l'ensemble du Sud-Est, elle a été modifiée dans des régions bien localisées [12].

Avec cette meilleure estimation de $J$, on a recalculé le $\hat{Q} I X A_{10}$ estimé par la méthode SOCOSE et on a procédé à une nouvelle régression linéaire entre le débit de crue décennale " observé » et le débit de crue décennale " estimé par SOCOSE régionalisée " :

$$
\ln \left(Q I X A_{10}\right)=1,05 \ln \left(\hat{Q} I X A_{10}\right)-0,258
$$

avec

$r=0,923$

$r^{2}=0,85$

$s_{\text {In }}=0,57$

Conclusion :

Le réglage, a posteriori, de la constante dans l'expression de l'interception potentielle $J$, améliore la prédétermination du débit instantané de crue décennale $Q I X A_{10}$, mais n'augmente pas pour autant sa fiabilité.

Les expressions permettant d'estimer la durée caractéristique de crue décennale $D$ d'un bassin versant, à partir de ses caractéristiques physiques, doivent pouvoir être améliorées régionalement à condition de disposer d'un échantillon de valeurs observées suffisamment représentatif de la région Sud-Est.

Cette méthode SOCOSE, outre l'estimation de la durée caractéristique de crue $D$, présente l'intérêt d'une approche différente, plus déterministe, que celle de la méthode CRUPEDIX uniquement statistique.

Pour l'estimation des débits instantanés de crues décennales, on ne peut done que recommander d'utiliser les deux méthodes en parallèle, lorsque l'on dispose des données nécessaires, et de confrônter les résultats qu'elles fournissent.

\section{Conclusion}

L'objet de cette note étant d'abord opérationnel, on conclut par des recommandations pratiques.

\section{a) Pour l'estimation des débits instantanés de crues de fréquence décennale}

Cette synthèse présente plusieurs approches possibles pour une estimation rapide, mais sommaire :

al) Une carte des débits pseudospécifiques, interpolée sur l'ensemble du Sud-Est.

Cette carte permet d'estimer des débits spécifiques pour une surface donnée. L'importance du facteur "aire considérée " pour ce type de calcul est illustrée par 3 cartes de débits spécifiques estimés pour des surfaces de $1 \mathrm{ha}, 1 \mathrm{~km}^{2}$, $100 \mathrm{~km}^{2}$.

a2) Deux formules d'estimation de type CRUPEDIX, suivant que l'on connaît ou non le module interannuel $Q A$.
Les formules sont accompagnées d'un intervalle de confiance à $70 \%$ et d'un intervalle de confiance à $90 \%$ indiquant les limites, en précision, de ces formules.

Pour chacune de ces formules, une carte en couleur indique également la grandeur d'un facteur multiplicateur de correction régional. Il pourra servir, moyennant quelques précautions, à améliorer l'estimation du débit instantané de crue de fréquence décennale.

Les seules variables explicatives utilisées pour estimer le débit instantané maximal décennal, outre le module interannuel spécifique, sont faciles à obtenir: il s'agit de la surface $S$ du bassin versant et de la pluie journalière maximale décennale $P J X A_{10}$.

D'autres variables ont été testées, mais elles n'apportaient pas d'explication supplémentaire significative au sens statistique (test de Student sur les coefficients de corrélation partielle). Elles n'ont donc pas été retenues. Leurs protocoles de mesure n'ayant pas toujours été valable (cf. [7] et [8]), certaines d'entre elles pourraient être re-testées à l'avenir.

a3) La formule SOCOSE nationale, dont les résultats sont validés sur la région Sud-Est.

Les estimations de la durée caractéristique de crue décennale $D$ et de l'interception potentielle $J$ n'ont pas pu être améliorées. On a cependant réalisé un calage régional de la formule nationale en définissant un coefficient régional de correction des estimations, qui a été introduit dans la constante de la formule d'estimation de $J$.

Ce calage, s'il améliore la prédétermination du débit instantané maximal décennal sur les bassins versants testés, n'en augmente pas pour autant sa fiabilité.

Un certain nombre de variables ont été testées dans l'espoir d'améliorer l'estimation de $D$ et de $J$, mais aucune n'apportait d'explication supplémentaire significative au sens statistique, et les formules nationales ont été conservées (sauf pour la valeur de la constante dans l'expression de l'interception potentielle $J$ ).

\section{b) Pour l'estimation des débits instantanés de crues de fréquence biennale}

S'inspirant de la méthode CRUPEDIX, deux formules de type CRUPEDEUX ont été calées, sur 208 et 189 bassins versants, pour estimer rapidement le débit instantané maximal biennal.

Pour chacune de ces formules, une carte en couleur indique également la grandeur d'un facteur multiplicateur de correction régional. Il pourra servir, toujours avec précautions, à améliorer l'estimation du débit instantané de crue biennal.

Les variables explicatives sont équivalentes à celles des formules de type CRUPEDIX : $S, P J X A_{2}$, et $Q A / S$.

Là encore, on n'a pas pu, pour l'instant, en déterminer d'autres qui apportent une contribution significative au sens statistique (voir les rapports intermédiaires). Les variables attribuées aux bassins versants sont essentiellement les mêmes que pour l'estimation du débit décennal (mêmes remarques sur leur précision, qui peut être améliorée). 


\section{c) En conclusion}

Toutes les formules présentées ici possèdent un intervalle de confiance encore relativement large, qu'elles soient purement statistiques comme celles produites par CRUPEDIX et CRUPEDEUX, ou plus déterministes comme celles produites par la méthode SOCOSE.

Certains point nécessiteraient des études complémentaires, en fonction des données disponibles.

En conséquence, lors d'une utilisation pratique, il est donc recommandé de procéder à un calage local de ces méthodes à l'aide de station(s), proche(s) du site étudié.

L'application de ces formules aux petits bassins torren- tiels est pour l'instant aventureuse. Un effort de récolte de données, sur ces petits bassins amonts, serait nécessaire pour tenter de produire des formules adaptées. Une autre solution, pour ces bassins torrentiels, est le recours à des modèles plus déterministes, mais il faut alors mieux connaître les pluies.

Enfin, on rappelle que toutes ces formulations sommaires ne doivent servir que de première estimation, ou de règle de transfert d'un bassin observé au site étudié.

Les cartes de débits pseudospécifiques, ou de facteurs de correction, peuvent aider à un tel transfert, car elles esquissent des similitudes régionales, d'un bassin à un autre.

\section{Références bibliographiques}

[1] Synthèse nationale sur les crues des petits bassins versants. 4 fascicules. SRAE, Min. Agric., CEMAGREF, 1980/86.

[2] Sakout S. et Guilbot A. : Essai d'estimation des débits de pointe de crue à partir des caractéristiques physiques et climatiques des bassins versants en région méditerranéenne : le cas du Languedoc Roussillon. Hydrologie Continentale, Vol. 3, $\mathrm{n}^{\circ} 1,1988$.

[3] Flow Regimes from Experimental and Network Data (FREND) UNESCO, Institute of Hydrology (NERC), 1989.

[4] Choisnel E. et Pagen D. : Les climats de la France. La Recherche : Météo, numéro supplément au $n^{\circ} 201$, juillet/août 1988.

[5] Analyse des fortes pluies de 1 à 10 jours sur 300 postes du Sud-Est de la France. CEMAGREF et Météorologie, 1982.

[6] Calcul du module interannuel en application de l'article $410 \mathrm{du}$ code rural. Note du Ministère de l'Environnement, 1987.

[7] Crues des petits bassins du Sud-est de la France. Estimation sommaire du débit instantané décennal. Rapport intermédiaire $n^{\circ} 1$. CEMAGREF, 1983.

[8] Contribution à l'amélioration de l'estimation des crues dans les petits bassins versants du Sud-Est. Rapport intermédiaire $\mathrm{n}^{\circ} 2$. Mémoire ENITRTS de Bernard Laget, 1984.

[9] Crues des petits bassins du Sud-Est de la France $\left(<1000 \mathrm{~km}^{2}\right)$. Estimation sommaire du débit instantané décennal. Rapport intermédiaire $\mathrm{n}^{\circ} 3$ (suite du rapport intermédiaire $\left.\mathrm{n}^{\circ} 1\right)$. CEMAGREF, 1983.
[10] Crues des petits bassins du Sud-Est de la France. Essai de formalisation régionale d'une méthode d'estimation sommaire du débit instantané biennal. Rapport intermédiaire $n^{\circ} 4$. CEMAGREF, 1985.

[11] Contribution à l'amélioration de l'estimation des crues dans les petits bassins versants du Sud-Est de la France, de taille inférieure à $100 \mathrm{~km}^{2}$. Rapport intermédiaire $\mathrm{n}^{\circ} 6$. Info Technique CEMAGREF, cahier $60, n^{\circ} 3,1985$.

[12] Crues des petits bassins de moins de $1000 \mathrm{~km}^{2}$ du Sud-Est de la France. Prédétermination du débit instantané décennal. Essai de régionalisation de la méthode SOCOSE nationale. Rapport intermédiaire $\mathrm{n}^{\circ} 7$. CEMAGREF, 1986.

[13] BERTIN J. : Le graphique et le traitement graphique de l'information. Flammarion, 1977.

[14] CUENIN R. : La cartographie générale. Tome 1 : notions générales et principes d'élaboration. Edition Eyrolles, 1972.

[15] GalÉA G., Lıxın J. (1990) : Modèles descriptifs synthétiques des connaissances régionales en crue : représentativité spatiale et domaines de validité. DEA ULPS/ENITRTS Strasbourg.

[16] Péguy C.P. et al. (1970 et s.) : Cartes climatiques détaillées de la France. ER - 30 CNRS et Ophrys. Grenoble-Gap.

[17] CEMAGREF, LAMA et al. (1989): Analyse des crues des petits bassins versants du Sud-Est de la France. Estimations sommaires des débits décennaux et biennaux. Cartes de Synthèse. CEMAGREF Lyon. 


\section{Notations utilisées}

\begin{tabular}{|c|c|}
\hline$Q I X A_{10}$ & $\begin{array}{l}=\text { débit instantané maximal annuel décenna } \\
\left(\mathrm{en}^{3} / \mathrm{s}\right) \text {. }\end{array}$ \\
\hline$Q I X A_{2}$ & $\begin{array}{l}=\text { débit instantané maximal annuel bienna } \\
\left(\mathrm{en}^{3} / \mathrm{s}\right) \text {. }\end{array}$ \\
\hline$Q A$ & $=$ module interannuel $\left(\mathrm{en}^{3} / \mathrm{s}\right)$ \\
\hline$D$ & $\begin{array}{l}=\text { durée caractéristique de crue pour } \\
\text { fréquence décennale, pendant laquelle } \\
\text { débit dépasse } Q I X A_{10} / 2 \text {, toujours pour } \\
\text { seule pointe principale de crue. }\end{array}$ \\
\hline$P J X A_{10}$ & $\begin{array}{l}\text { = pluie locale journalière maximale annuell } \\
\text { décennale }(\mathrm{en} \mathrm{mm}) \text {. }\end{array}$ \\
\hline$P J X A_{2}$ & $\begin{array}{l}=\text { pluie locale journalière maximale annuell } \\
\text { biennale }(\mathrm{en} \mathrm{mm} \text { ). }\end{array}$ \\
\hline$P A$ & $\begin{array}{l}\text { = précipitation annuelle moyenne de bassir } \\
\text { (en } \mathrm{mm} \text { ). }\end{array}$ \\
\hline$T A$ & $\begin{array}{l}=\text { température annuelle moyenne du bassin } \\
\text { réduite au niveau de la mer }\left(\mathrm{en}^{\circ} \mathrm{C}\right) \text {. }\end{array}$ \\
\hline
\end{tabular}
$S$
$J$
$=$ surface du bassin versant $\left(\mathrm{en}^{\mathrm{k}} \mathrm{km}^{2}\right)$.
$=$ interception potentielle (en $\mathrm{mm}$ ).
$=$ coefficient régional de correction des formules d'estimation (sans dimension).
= ce chapeau indique que la variable concer- née est " estimée " (formules) et non " obser- vée " (statistiques sur observations).
ln $\quad=$ logarithme népérien (naturel).
** $\quad=$ symbole "exposant $»$.
$\{x\}=$ ces accolades signifient que $x$ est $«$ esti- mée " et non " observée ".

\section{Sigles}

DTG : Division technique générale (Grenoble) d'EdF.

LAMA : Laboratoire de la montagne alpine (Grenoble).

RTM : Restauration des terrains en montagne.

SN : Service de la Navigation.

SRAE : Service régional d'aménagement des eaux.

\section{ANNEXE}

\section{Variables analysées}

(des combinaisons de ces variables ont aussi été étudiées).

A) Liste des variables étudiées dans [7], [9] et [10]

$Q J X A_{10}:$ Débit journalier maximal dans l'année, de fréquence décennale.

$Q I X A_{10} \quad$ : Débit instantané maximal dans l'année, de fréquence décennale.

$D \quad$ : Durée caractéristique de crue pour la fréquence décennale $(h)$; valeur observée (mesurée sur des hydrogrammes) selon les définitions du fascicule 2 de la Synthèse Nationale: la méthode SOCOSE, janvier 1980.

$S \quad$ : Surface, en $\mathrm{km}^{2}$.

$Z \quad$ : Altitude de la station de contrôle (m).

$Z X B \quad$ : Altitude du point culminant du bassin (m).

$L \quad$ : Longueur du thalweg le plus long $(\mathrm{km})$, (éventuelle partie amont glaciaire incluse).

$P A B \quad$ : Pluie annuelle (moyenne interannuelle) de bassin $(\mathrm{mm})$.

$P J X A_{10}$ : Pluie journalière maximale décennale $(\mathrm{mm})$ locale en un lieu (poste réel) supposé représentatif du bassin.

Ces pluies sont centrées sur les intensités maximales ; (cf. Synthèse Sud-Est sur 300 postes pluviométriques).

$T A \quad$ : Température annuelle (moyenne interannuelle) ramenée au niveau de la mer (en ${ }^{\circ} \mathrm{C}$ ).

$B \quad$ : Coefficient de MONTANA (ordinaire : $P=A t^{1-B}$ ) sur les pluies de durée " DUR ». $1 \leqslant$ DUR $\leqslant n$ jours avec $2 \leqslant n \leqslant 10$ selon les cas.

ZXT : Altitude maximale du thalweg le plus long $(\mathrm{m})$ (y compris si l'amont du thalweg est glaciaire).

$S G \quad: \%$ de surface du bassin en glaciers.

$S L M \quad: \%$ de surface du bassin en lacs et marais. ajust. statistiques sur observations 


\section{B) Liste des variables étudiées dans [8]}

(en sus de celles de la liste A).

SF $\quad: \%$ de Surface du bassin en Forêt ;

$S B \quad: \%$ de Surface du bassin en Broussaille;

FRETA : FREquence des TAlwegs.

\section{C) Quelques caractéristiques hydrologiques}

(autres que de crues annuelles présentées dans la liste A).

\begin{tabular}{|c|c|}
\hline PMCM & $\begin{array}{l}\text { Précipitations Mensuelles } \quad \text { Cumulées } \\
\text { Médianes, en mm }\end{array}$ \\
\hline$Q A$ & Module interAnnuel, en $\mathrm{m}^{3} / \mathrm{s}$ \\
\hline$Q M N A_{2}$ & $\begin{array}{l}\text { : Débit d'étiagē Mensuel médian, en } \mathrm{m}^{3} / \mathrm{s} \\
\text { (étiage }=\text { miNimal } ; \text { médian }=2 \text { ans). }\end{array}$ \\
\hline$Q M X A_{2}$ & $\begin{array}{l}\text { : Débit Mensuel maXimal médian, en } \mathrm{m}^{3} / \mathrm{s} \\
\text { (médian }=2 \text { ans). }\end{array}$ \\
\hline$Q N D$ & $\begin{array}{l}\text { Débit du mois d'étiage de l'année } 1975 \text {, en } \\
\mathrm{m}^{3} / \mathrm{s} \text { (étiage }=\text { miNimal). }\end{array}$ \\
\hline$Q I X N_{2}$ & $\begin{array}{l}\text { Débit Instantané maXimal médian de la } \\
\text { saison N-eigeuse (mai à juillet), en } \mathrm{m}^{3} / \mathrm{s} \\
\text { (médian }=2 \text { ans). }\end{array}$ \\
\hline$Q I X P_{2}$ & $\begin{array}{l}\text { Débit Instāntané maXimal médian de la } \\
\text { saison P̄luviale (août à avril), en } \mathrm{m}^{3} / \mathrm{s} \\
\text { (médian }=2 \text { ans). }\end{array}$ \\
\hline
\end{tabular}

D) Quelques caractéristiques hydrauliques au niveau de la station de jaugeage

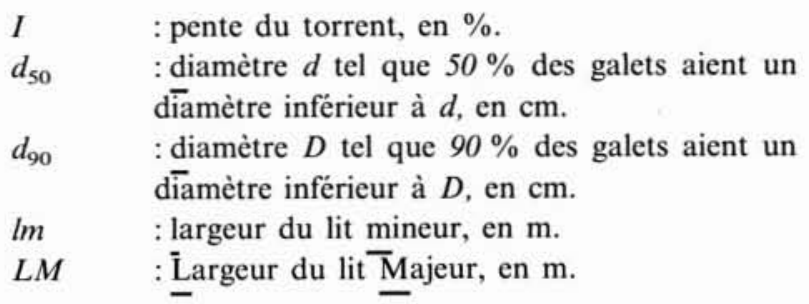

E) Courbes hypsométriques de 83 bassins versants

\begin{tabular}{|c|c|}
\hline $\begin{array}{l}\text { F) Liste des } \\
\text { versants }\end{array}$ & paramètres caractérisant le relief des bassins \\
\hline$l$ & : largeur du rectangle équivalent. \\
\hline$L$ & : Longueur du rectangle équivalent. \\
\hline$D$ & 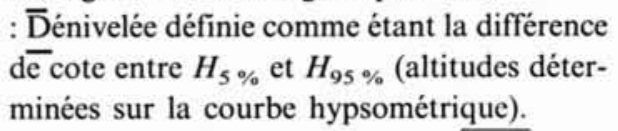 \\
\hline $\begin{array}{l}D_{s} \\
\text { TAYSLO }\end{array}$ & $\begin{array}{l}\text { Dénivelée spécifique }\left(D_{s}=D \sqrt{1 / L)}\right. \\
: \text { pente du TAlweg principal. }\end{array}$ \\
\hline$H_{50} \%$ & $\begin{array}{l}\text { : altitude médiane du bassin versant, déter- } \\
\text { minée à partir de la courbe hypsométrique. }\end{array}$ \\
\hline$\sum_{i} S_{i} Z_{i}$ & $\begin{array}{l}\text { : paramètre résultant d'un planimétrage sur } \\
\text { la courbe hypsométrique }\left(S_{i} \text { est la superficie }\right. \\
\left.\text { du bassin à l'altitude } Z_{i}\right) \text {. }\end{array}$ \\
\hline
\end{tabular}

\begin{tabular}{|c|c|}
\hline & $\begin{array}{l}\text { Géologie des barrages et des retenues de } \\
\text { petites dimensions - Danielle Lautrin } \\
\text { Géologie appliquée à la construction de barrages de moins de } \\
20 \text { mètres. } \\
1990,144 \text { pages, illustrations - Prix : } 200 \text { F TTC }\end{array}$ \\
\hline \multicolumn{2}{|c|}{$\begin{array}{l}\text { La forêt et ses ennemis - J.F. Abgrall et A. Soutrenon } \\
88 \text { fiches décrivent les principaux insectes ravageurs, champignons et maladies des forêts. } \\
\text { S'adresse à tous les gestionnaires de la forêt, à l'enseignement et à tous ceux qui se passionnent } \\
\text { pour la forêt et la protection de la nature. } \\
1991,3 \text { e édition, } 21 \times 29,7, \text { relié, } 400 \text { pages, } 485 \text { photos - Prix : } 340 \mathrm{~F} \mathrm{TTC}\end{array}$} \\
\hline & (EF-DICOVA BP 22, 92162 ANTONY CEDEX Tél. : (1) 40966132 \\
\hline
\end{tabular}

